\title{
Pacific
}

Journal of

Mathematics

\section{SPLITTING CRITERIA FOR VECTOR BUNDLES ON HIGHER-DIMENSIONAL VARIETIES}

\author{
PARSA BAKHTARY
}




\title{
SPLITTING CRITERIA FOR VECTOR BUNDLES ON HIGHER-DIMENSIONAL VARIETIES
}

\author{
PARSA BAKHTARY
}

\begin{abstract}
We generalize Horrocks' criterion for the splitting of vector bundles on projective space by establishing an analogous splitting criterion for vector bundles on a class of smooth complex projective varieties of dimension $\geq 4$, over which every extension of line bundles splits.
\end{abstract}

\section{Introduction}

Algebraic geometry has a rich history of studying when a vector bundle over a projective space splits, that is, when it is isomorphic to a direct sum of line bundles. Grothendieck [1957] first used cohomological methods in sheaf theory to prove his celebrated theorem which says that every vector bundle over $\mathbb{P}^{1}$ splits as a direct sum of line bundles. This was followed by Horrocks' [1964] famous criterion, which announced that a vector bundle on $\mathbb{P}^{n}$ for $n \geq 3$ splits if and only if its restriction to a hyperplane $H=\mathbb{P}^{n-1} \subset \mathbb{P}^{n}$ splits.

Soon after came the notoriously difficult conjectures of Hartshorne [1974], which state that all vector bundles of rank 2 on $\mathbb{P}^{n}$ with $n \geq 7$ must split, though over $\mathbb{C}$ no nonsplitting (indecomposable) 2-bundle over $\mathbb{P}^{5}$ is known. Over the complex numbers, the Horrocks-Mumford [1973] bundle is the only nonsplitting 2-bundle known on $\mathbb{P}^{4}$, and its existence is far from obvious. (There are rank- 2 indecomposable bundles on $\mathbb{P}^{5}$ in characteristic 2 [Tango 1976] and on $\mathbb{P}^{4}$ in any positive characteristic different from the Horrocks-Mumford bundle [Kumar 1997].)

There has been a formidable body of work dedicated to finding splitting criteria and constructing indecomposable bundles over projective space, and the wellknown but out-of-print book by Okonek, Schneider, and Spindler [1980] gives an excellent survey of progress made in this direction up until its publication. There has also been much work since then, with many notable results [Ancona et al. 1994; Ballico 1995; Kumar et al. 2002, 2003; Malaspina 2008; Ran 1983; Sumihiro and Tagami 2001; Zak 1993]. In addition to splitting criteria for $r$-bundles on multiprojective spaces [Ballico and Malaspina 2009; Costa and Miró-Roig 2005],

Partially supported by a KFUPM FT grant.

MSC2000: 14J60, 14F05.

Keywords: splitting, vector bundle, Horrocks' criterion, Grothendieck-Lefschetz theorem. 
cones over rational normal curves [Ballico 2008] and blowings up of the plane [Ballico and Malaspina 2008], extensions of Horrocks' criterion to Grassmannians and quadrics have been established [Ancona et al. 1994; Ottaviani 1989]. Furthermore, the splitting of 2-bundles on hypersurfaces in $\mathbb{P}^{4}$ and $\mathbb{P}^{5}$ has been studied [Madonna 1998; Chiantini and Madonna 2004] and generalized in [Kumar et al. 2007a] and [Kumar et al. 2007b], which show respectively that arithmetically Cohen-Macaulay 2-bundles on general hypersurfaces of degree $\geq 6$ in $\mathbb{P}^{4}$ and on general hypersurfaces of degree $\geq 3$ in $\mathbb{P}^{5}$ must split. Further, notions such as uniform vector bundles have been generalized to Fano manifolds [Wiśniewski 2002].

However, to the author's knowledge, the literature lacks a study of when a Horrocks' type criterion occurs on arbitrary smooth projective varieties. The spirit in which we pursue this question is similar to that of Horrocks': when can we reduce the splitting of a vector bundle $E$ on a smooth projective variety $X$ to the splitting of the restriction $E_{\mid Y}$ for a suitable proper closed subscheme $Y \subset X$ ? Horrocks showed that as soon as the dimension of a projective space is at least three, the splitting of a vector bundle on that projective space is equivalent to the splitting of its restriction to a hyperplane. In this scenario, the restriction map $\operatorname{Pic}\left(\mathbb{P}^{n}\right) \stackrel{\sim}{\rightarrow} \operatorname{Pic}(H)$ is an isomorphism, so the line bundles on the subscheme $H$ are precisely those coming from $\mathbb{P}^{n}$, no more, no less. Thus if $E_{\mid H}$ splits, we already have a suitable candidate on $\mathbb{P}^{n}$ that $E$ ought to be isomorphic to, should it split. The dimension of the hyperplane being at least 2 is crucial, since any nonsplitting bundle must split when restricted to a line $\mathbb{P}^{1}$ by Grothendieck's theorem.

We remedy this issue for higher-dimensional varieties using the GrothendieckLefschetz theorem on Picard groups (see [Hartshorne 1970] for an exposition), which says that if $X$ is a smooth complex projective variety of dimension $n \geq 4$, then given any ample effective divisor $D$ (not necessarily reduced) on $X$, the natural restriction map $\operatorname{Pic}(X) \rightarrow \operatorname{Pic}(D)$ is an isomorphism. In this way, we ensure that the line bundles on our divisor $D$ are precisely those coming from $X$, as in Horrocks' situation with projective space. Then, we assume that $E_{\mid D}$ splits over $D$, and our task is to try to lift a given isomorphism $E_{\mid D} \stackrel{\sim}{\rightarrow} \bigoplus L_{i \mid D}$ to one on $X$, or to find the obstruction to such a lifting.

Though this lifting does not exist in general, it can be found on a certain class of varieties. We call a scheme $X$ a Horrocks scheme if $H^{1}(X, L)=H^{2}(X, L)=0$ for every line bundle $L$ on $X$. A Horrocks scheme is like projective $n \geq 3$ space in the sense that every extension of line bundles splits. Here a criterion of Horrocks type holds.

Our main result is the following theorem.

Theorem 1.1. Let $X$ be a smooth complex projective Horrocks variety of dimension $n \geq 4$. A vector bundle $E$ on $X$ splits if and only if $E_{\mid D}$ splits over $D$, where $D$ is an ample effective divisor on $X$. 


\section{Preliminaries}

Nearly all of the results we use are familiar to a seasoned student of algebraic geometry, and can be found throughout [Hartshorne 1977]. Throughout this paper we will work over $\mathbb{C}$. In this section we mention some of the deeper theorems that are relevant to the proofs in the next section. We first state Horrocks' criterion in its full form.

Theorem 2.1 (Horrocks). Let $E$ be a rank $r$ vector bundle on $\mathbb{P}^{n}$. Then $E$ splits if and only if $H^{i}\left(\mathbb{P}^{n}, E(k)\right)=0$ for every $k \in \mathbb{Z}$ and every $i$ with $0<i<n$.

Proof. See [Horrocks 1964] or [Okonek et al. 1980].

Corollary 2.2 (Horrocks). Let $E$ be a rank $r$ vector bundle on $\mathbb{P}^{n}$ with $n \geq 3$. Then $E$ splits if and only if its restriction $E_{\mid H}$ to a hyperplane $H \cong \mathbb{P}^{n-1} \subset \mathbb{P}^{n}$ splits.

Thus, by induction it suffices to find a plane $P \cong \mathbb{P}^{2} \subset \mathbb{P}^{n}$ such that $E_{\mid P}$ splits.

Recall the formal completion of $X$ along a closed subscheme $Z$ defined by the ideal $\Phi \subset O_{X}$ is the ringed space $\left(\hat{X}, O_{\hat{X}}\right)$ whose topological space is $Z$ and whose structure sheaf is $\lim \left(0_{X} / \mathscr{I}^{m}\right)$. Given a coherent sheaf $\mathscr{F}$ on $X$, we define the completion of $\mathscr{F}$ along $Z$, denoted by $\hat{\mathscr{F}}$, to be the sheaf $\lim \left(\mathscr{F}_{F} / \mathscr{I}^{m} \mathscr{F}\right)$ on $Z$, which has the natural structure of an $\hat{O}_{\hat{X}}$-module.

Our most important gadget is the Grothendieck-Lefschetz theorem on Picard groups. We do not require the most general version.

Theorem 2.3 (Grothendieck). Let D be an ample, effective divisor (not necessarily reduced) on a smooth complex projective variety $X$ of dimension $n \geq 4$. Then the natural restriction map $\operatorname{Pic}(X) \rightarrow \operatorname{Pic}(D)$ is an isomorphism.

Proof. See [Grothendieck 1968] or [Hartshorne 1970].

In conjunction with [Hartshorne 1977, Exercise II.9.6], we have the following chain of natural isomorphisms for any positive integer $m$ :

$$
\operatorname{Pic}(X) \stackrel{\sim}{\rightarrow} \operatorname{Pic}(\hat{X}) \stackrel{\sim}{\rightarrow} \lim \operatorname{Pic}(m D) \stackrel{\sim}{\rightarrow} \operatorname{Pic}(m D) \stackrel{\sim}{\rightarrow} \operatorname{Pic}(D)
$$

whose composition is the natural restriction map isomorphism mentioned in the theorem.

\section{Arbitrary varieties}

We first study the splitting behavior of a vector bundle restricted to the formal completion of a projective manifold along an ample effective divisor, and show this is equivalent to the splitting of the bundle itself.

Proposition 3.1. Let $X$ be a smooth complex projective variety of dimension $n \geq 4$, and let $E$ be a vector bundle of rank $r$ on $X$. Then $E$ splits over $X$ if and only if $\hat{E}$ splits over $\hat{X}$, where $\hat{X}$ is the completion of $X$ along an ample effective divisor $D$. 
Proof. For the forward direction, suppose $E$ splits over $X$, that is, $E \cong \bigoplus L_{i}$. Then $\hat{E} \cong \bigoplus \hat{L}_{i}$, and each $\hat{L}_{i}$ is a line bundle on $\hat{X}$.

For the other direction, suppose $\hat{E}$ splits as a direct sum of line bundles on $\hat{X}$. Then since

$$
\operatorname{Pic}(X) \cong \operatorname{Pic}(\hat{X}) \cong \lim _{\longleftarrow} \operatorname{Pic}(m D) \cong \operatorname{Pic}(m D) \cong \operatorname{Pic}(D),
$$

we must have $\hat{E} \cong \bigoplus \hat{L}_{i}$, for some line bundles $L_{i}$ on $X$. Set $F:=\bigoplus L_{i}$. Tensoring the short exact sequence

$$
0 \rightarrow \mathrm{O}_{X}(-m D) \rightarrow \mathrm{O}_{X} \rightarrow \mathrm{O}_{m D} \rightarrow 0
$$

with $F^{*} \otimes E \cong \mathscr{H o m}(F, E)$ we obtain

$$
0 \rightarrow \mathrm{O}_{X}(-m D) \otimes F^{*} \otimes E \rightarrow F^{*} \otimes E \rightarrow F_{\mid m D}^{*} \otimes E_{\mid m D} \rightarrow 0 .
$$

Choosing $m \gg 0$ and using Serre duality plus the fact that $O_{X}(D)$ is ample, we can force $H^{1}\left(X, O_{X}(-m D) \otimes F^{*} \otimes E\right)=0$, and we get a surjection

$$
\operatorname{Hom}(F, E) \rightarrow \operatorname{Hom}\left(F_{\mid m D}, E_{\mid m D}\right) .
$$

We can lift a given isomorphism $\varphi: F_{\mid m D} \stackrel{\sim}{\rightarrow} E_{\mid m D}$ (this is just our original isomorphism $\hat{F} \stackrel{\sim}{\rightarrow} \hat{E}$ restricted to a finite thickening $m D$ ) to a homomorphism $\psi: F \rightarrow E$ on $X$. The bundles $E$ and $F$ have the same rank and first Chern class, the latter because $\mathcal{O}_{m D} \cong \operatorname{det} E_{\mid m D} \otimes \operatorname{det} F_{\mid m D}^{*} \cong \mathcal{O}_{X}\left(c_{1}(E)-c_{1}(F)\right)_{\mid m D}$ implies that $\mathcal{O}_{X}\left(c_{1}(E)\right) \cong \mathcal{O}_{X}\left(c_{1}(F)\right)$ on $X$ since the restriction map $\operatorname{Pic}(X) \stackrel{\sim}{\rightarrow} \operatorname{Pic}(m D)$ is an isomorphism. Thus,

$\operatorname{det} \psi \in \operatorname{Hom}(\operatorname{det} F, \operatorname{det} E) \cong H^{0}\left(X, O_{X}\left(c_{1}(E)-c_{1}(F)\right)\right) \cong H^{0}\left(X, O_{X}\right) \cong \mathbb{C}$

is a nonzero constant since $\psi$ restricts to an isomorphism on $m D$. Hence $\psi$ is invertible.

Remark 3.2. This proposition illustrates that, in the setting above, if $E_{\mid D}$ splits on a sufficiently positive divisor $D$ on $X$, then $E$ must split over $X$. One possible approach is to make positivity assumptions on $D$ in terms of the Chern classes of $E$.

\section{Horrocks schemes}

We begin with the definition of a splitting scheme and a Horrocks scheme, which capture a cohomological feature of line bundles on projective spaces, and we give some examples.

Definition 4.1. A scheme $X$ is called a splitting scheme if $H^{1}(X, L)=0$ for any line bundle $L$ on $X$. Equivalently, $\operatorname{Ext}^{1}(L, M)=0$ for any line bundles $L, M$ on $X$, that is, any extension of line bundles splits. 
Definition 4.2. A scheme $X$ is called a Horrocks scheme if $H^{i}(X, L)=0$ for $i=1,2$ and any line bundle $L$ on $X$.

Remark 4.3. For a smooth projective variety to be a splitting scheme, its dimension must be at least two. For a curve, we would have by Serre duality that

$$
H^{1}\left(C, L^{-1}\right) \cong H^{0}\left(C, L \otimes \omega_{C}\right) \neq 0
$$

for a sufficiently ample line bundle $L$ on $C$. Similarly a smooth projective variety must be of dimension at least three in order to be a Horrocks scheme, and for threefolds these notions are equivalent.

The following are examples of Horrocks schemes. It is clear how to adjust the constructions to obtain splitting schemes.

Example 4.4. Clearly projective space $\mathbb{P}^{n}$ with $n \geq 3$ is a Horrocks scheme.

Example 4.5. If $X$ and $Y$ are projective Horrocks varieties, then

$$
\operatorname{Pic}(X \times Y) \cong \operatorname{Pic}(X) \times \operatorname{Pic}(Y)
$$

since $H^{1}\left(X, O_{X}\right)=0$; see [Hartshorne 1977, Exercise III.12.6]. Using the Künneth formula, we see that the fiber product $X \times Y$ remains a Horrocks variety. In particular, multiprojective spaces $P=\mathbb{P}^{n_{1}} \times \cdots \times \mathbb{P}^{n_{k}}$ are Horrocks schemes if each $n_{i} \geq 3$.

Example 4.6. Weighted projective spaces $W=\mathbb{P}\left(a_{0}, \ldots, a_{n}\right)$ with $n \geq 3$ are singular Horrocks schemes; see [Dolgachev 1982, § 2].

Example 4.7. Any global complete intersection $X \subset \mathbb{P}^{N}$ of dimension $n \geq 3$ is necessarily a Horrocks scheme, since the Lefschetz theorem on Picard groups implies that $\operatorname{Pic}(X) \cong \mathbb{Z}$ and we know that $H^{i}\left(X, O_{X}(m)\right)=0$ for $0<i<\operatorname{dim} X$ and every $m \in \mathbb{Z}$; see [Hartshorne 1970, Chapter IV, § 3] and [Hartshorne 1977, Exercise III.5.5(c)].

Example 4.8. Any Grassmannian $G$ of dimension $n \geq 3$ is a Horrocks scheme. Since $\operatorname{Pic}(G) \cong \mathbb{Z}$, let $\mathcal{O}(1)$ denote the ample generator. Then $H^{i}(G, \mathcal{O}(m))=0$ for $i=1,2$ and $m<0$ by Kodaira vanishing, and for $m \geq 0$, we have

$$
H^{i}(G, O(m)) \cong H^{n-i}\left(G, O(-m) \otimes \omega_{G}\right)=0
$$

for $i=1,2$ by Serre duality, Kodaira vanishing, and the fact that $G$ is Fano.

Example 4.9. Let $X$ be a smooth projective Horrocks variety. Let $E$ be a direct sum of $r \geq 4$ line bundles on $X$, and consider the projectivized space bundle

$$
P:=\mathbb{P}(E) \stackrel{\pi}{\rightarrow} X,
$$

where $\mathbb{P}(E)=\operatorname{Proj}(\operatorname{Sym}(E))$. We claim that $P$ is a Horrocks scheme as well. We already know that $P$ is a smooth projective variety with $\operatorname{Pic}(P) \cong \mathbb{Z} \cdot 0_{P}(1) \oplus \operatorname{Pic}(X)$. 
Thus, any line bundle on $P$ is isomorphic to one of the form $M:=\mathscr{O}_{P}(m) \otimes \pi^{*} L$, where $m \in \mathbb{Z}$ and $L$ is a line bundle on $X$. Since the fibers of $\pi$ are all isomorphic to $\mathbb{P}^{r-1}$ with $r-1 \geq 3$, we have

$$
R^{i} \pi_{*} M=0 \text { for } i=1,2 .
$$

For $m<0$ we have $\pi_{*} \hat{O}_{P}(m)=0$, and for $m \geq 0$ we have $\pi_{*} \hat{O}_{P}(m)=S^{m}(E)$, which is each isomorphic to a direct sum of line bundles on $X$ since $E$ is a direct sum of line bundles on $X$. Since $X$ is a Horrocks scheme, we have

$$
H^{i}\left(X, \pi_{*} M\right)=H^{i}\left(X, S^{m}(E) \otimes L\right)=0 \quad \text { for } i=1,2 .
$$

The Leray spectral sequence implies immediately that

$$
H^{1}(P, M)=H^{1}\left(X, \pi_{*} M\right)=0,
$$

and it also follows that $H^{2}(P, M)=E_{\infty}^{0,2} \oplus E_{\infty}^{1,1} \oplus E_{\infty}^{2,0}$, where

$$
E_{2}^{p, q}=H^{p}\left(X, R^{q} \pi_{*} M\right)
$$

abuts to $H^{p+q}(P, M)$. But each $E_{2}^{p, q}$ with $p+q=2$ is 0 by the vanishings above, hence the infinity pages vanish also. Thus $P=\mathbb{P}(E)$ is also a Horrocks scheme.

Remark 4.10. In the previous example, we do not actually need $E$ to be a direct sum of line bundles, provided it has a sufficiently short resolution by direct sums of line bundles. For example, on $\mathbb{P}^{n}$ the Euler sequence gives

$$
0 \rightarrow \mathrm{O} \rightarrow \mathcal{O}(1)^{\oplus(n+1)} \rightarrow T_{\mathbb{P}^{n}} \rightarrow 0,
$$

so we still have $H^{i}\left(\mathbb{P}^{n}, S^{m}\left(T_{\mathbb{P}^{n}}\right)(k)\right)=0$ for $i=1, \ldots, n-2$ and $m, k \in \mathbb{Z}$. Hence, $\mathbb{P}\left(T_{\mathbb{P} n}\right)$ is a splitting scheme for $n \geq 3$ and a Horrocks scheme for $n \geq 4$.

Proposition 4.11. Let $X$ be a smooth projective splitting variety of dimension $n \geq 4$. Then a vector bundle $E$ on $X$ splits if and only if

(1) there exists an ample effective divisor $D$ on $X$ such that $E_{\mid D}$ splits over $D$, and

(2) $H^{1}(X, E \otimes L)=0$ for every line bundle $L$ on $X$.

Proof. To show necessity, suppose that $E \cong \bigoplus L_{i}$ on $X$, where $L_{i}$ are line bundles on $X$. Then for any closed subscheme, $Z \subset X, E_{\mid Z} \cong \bigoplus L_{i \mid Z}$ splits as well, showing (1). The assumption that $X$ is a splitting scheme gives (2).

We show sufficiency. By (1) we have an isomorphism $\varphi: \bigoplus M_{i} \stackrel{\sim}{\rightarrow} E_{\mid D}$, where $M_{i}$ are line bundles on $D$. The Grothendieck-Lefschetz theorem tells us that the restriction map gives an isomorphism $\operatorname{Pic}(X) \stackrel{\sim}{\rightarrow} \operatorname{Pic}(D)$ of Picard groups. Lift each 
$M_{i}$ on $D$ uniquely (up to isomorphism) to $L_{i}$ on $X$ and set $F:=\bigoplus L_{i}$. Now tensor the short exact sequence

$$
0 \rightarrow \mathrm{O}_{X}(-D) \rightarrow \mathrm{O}_{X} \rightarrow \mathrm{O}_{D} \rightarrow 0
$$

with $F^{*} \otimes E \cong \mathscr{H o m}(F, E)$ and take cohomology to get an exact sequence

$$
H^{0}\left(X, F^{*} \otimes E\right) \rightarrow H^{0}\left(Y, F_{\mid D}^{*} \otimes E_{\mid D}\right) \rightarrow H^{1}\left(X, F^{*} \otimes E \otimes \mathcal{O}_{X}(-D)\right)=0,
$$

where the third vector space vanishes by (2). Thus we have a surjection

$$
\operatorname{Hom}(F, E) \rightarrow \operatorname{Hom}\left(F_{\mid D}, E_{\mid D}\right),
$$

so we may lift our isomorphism $\varphi$ to a homomorphism $\psi: F \rightarrow E$, and we claim that $\psi$ is an isomorphism. First, $E$ and $F$ have the same rank and the same first Chern class since $\mathrm{O}_{X}\left(c_{1}(E)-c_{1}(F)\right)_{\mid D} \cong \mathrm{O}_{D}$ implies that

$$
\operatorname{det}(F) \cong \mathrm{O}_{X}\left(c_{1}(F)\right) \cong \mathrm{O}_{X}\left(c_{1}(E)\right) \cong \operatorname{det}(E)
$$

because of the aforementioned isomorphism on Picard groups. Then $\psi$ induces $\operatorname{det}(\psi): \operatorname{det}(F) \rightarrow \operatorname{det}(E)$, which gives a section

$$
\operatorname{det}(\psi) \in H^{0}\left(X, \operatorname{det}(F)^{-1} \otimes \operatorname{det}(E)\right) \cong H^{0}\left(X, \mathscr{O}_{X}\left(c_{1}(E)-c_{1}(F)\right)\right) \cong H^{0}\left(X, \mathscr{O}_{X}\right) \cong \mathbb{C},
$$

which means that $\operatorname{det}(\psi)$ is multiplication by a constant. But $\operatorname{det}(\psi)$ restricts to an isomorphism $\operatorname{det}(\varphi)$ on $D$, hence it must be a nonzero constant and hence invertible, thus showing that $\psi$ is indeed an isomorphism.

Remark 4.12. From the proof, the sufficiency holds for arbitrary smooth projective varieties of dimension $\geq 4$, but the assumption that $X$ is a splitting scheme gives necessity.

The following proposition was pointed out to the author by N. Mohan Kumar.

Proposition 4.13. Let $X$ be a smooth projective variety of dimension $\geq 4$. The following are equivalent:

(1) $X$ is a Horrocks scheme.

(2) Every ample effective divisor $D$ on $X$ is a splitting scheme.

(3) There exists an ample effective divisor $D$ on $X$ which is a splitting scheme.

Proof. (1) $\Rightarrow(2)$ : Tensoring the short exact sequence

$$
0 \rightarrow \mathrm{O}_{X}(-D) \rightarrow \mathrm{O}_{X} \rightarrow \mathrm{O}_{D} \rightarrow 0
$$

with a line bundle $L$ on $X$ and taking cohomology, we get

$$
\cdots \rightarrow H^{1}(X, L) \rightarrow H^{1}\left(D, L_{\mid D}\right) \rightarrow H^{2}\left(X, L \otimes \mathrm{O}_{X}(-D)\right) \rightarrow \cdots .
$$


The outside terms vanish by assumption, and every line bundle on $D$ is isomorphic to one of the form $L_{\mid D}$, for some line bundle $L$ on $X$ by the Grothendieck-Lefschetz theorem. Hence $D$ is a splitting scheme by definition.

(2) $\Rightarrow$ (3): Trivial.

(3) $\Rightarrow(1)$ : Given $D \subset X$, an ample effective codimension-1 splitting scheme, consider the short exact sequence

$$
0 \rightarrow L \otimes \mathcal{O}_{X}((k-1) D) \rightarrow L \otimes \mathcal{O}_{X}(k D) \rightarrow L_{\mid D} \otimes \mathcal{O}_{X}(k D)_{\mid D} \rightarrow 0
$$

for every $k \in \mathbb{Z}$. Since $D$ is a splitting scheme, we have $H^{1}\left(D, L_{\mid D} \otimes \mathscr{O}_{X}(k D)_{\mid D}\right)=0$ for every $k \in \mathbb{Z}$, which after taking cohomology gives surjections

$$
H^{1}\left(X, L \otimes \mathcal{O}_{X}((k-1) D)\right) \rightarrow H^{1}\left(X, L \otimes \mathscr{O}_{X}(k D)\right)
$$

and injections

$$
H^{2}\left(X, L \otimes \mathcal{O}_{X}((k-1) D)\right) \hookrightarrow H^{2}\left(X, L \otimes \mathcal{O}_{X}(k D)\right)
$$

for every $k \in \mathbb{Z}$. Since $D$ is ample, we may take $k \ll 0$ and $k \gg 0$ respectively and use Serre vanishing to see that $H^{1}(X, L)=H^{2}(X, L)=0$ for any line bundle $L$ on $X$.

The following corollary is a generalization of Horrocks' criterion for projective $n \geq 3$ space.

Corollary 4.14. Let $X$ be a smooth projective Horrocks variety of dimension $n \geq 4$. $A$ vector bundle $E$ on $X$ splits if and only if its restriction $E_{\mid D}$ to an ample effective divisor $D \subset X$ splits.

Proof. We show the nontrivial direction. Assuming $E_{\mid D}$ splits over $D$, we see that condition (1) of Proposition 4.11 is immediately satisfied, so it suffices to check condition (2). Let $L$ be any line bundle on $X$, and tensor the short exact sequence

$$
0 \rightarrow \mathrm{O}_{X}(-D) \rightarrow \mathrm{O}_{X} \rightarrow \mathrm{O}_{D} \rightarrow 0
$$

with $E \otimes L \otimes \mathcal{O}_{X}(m D)$, and take cohomology to get

$$
\begin{aligned}
H^{1}\left(X, E \otimes L \otimes \mathcal{O}_{X}((m-1) D)\right) & \rightarrow H^{1}\left(X, E \otimes L \otimes \mathcal{O}_{X}(m D)\right) \\
& \rightarrow H^{1}\left(D, E_{\mid D} \otimes L_{\mid D} \otimes \mathcal{O}_{X}(m D)_{\mid D}\right)
\end{aligned}
$$

exact. By the previous proposition, $D$ is a splitting scheme, hence the third term vanishes for any $m \in \mathbb{Z}$ since $E_{\mid D}$ splits as a sum of line bundles. So we have surjections

$$
H^{1}\left(X, E \otimes L \otimes \mathcal{O}_{X}((m-1) D)\right) \rightarrow H^{1}\left(X, E \otimes L \otimes \mathcal{O}_{X}(m D)\right)
$$

for every $m \in \mathbb{Z}$. Taking $m \ll 0$ and using Serre duality, we can make the left hand side zero since $D$ is ample, and the surjections above imply that the cohomology 
must vanish for all integers $m$. In particular, taking $m=0$, we have $H^{1}(X, E \otimes L)=0$. Since $L$ was arbitrary, we have shown condition (2), which completes the proof.

There is a natural extension of this result using induction:

Corollary 4.15. Let $X$ be a smooth projective variety of dimension $n \geq 4$ such that $H^{i}(X, L)=0$ for $i=1, \ldots, d-1$ and every line bundle $L$, where $d \geq 3$. Suppose $H_{1}, \ldots, H_{n-d}$ are ample divisors such that $H_{1} \cap \cdots \cap H_{l}$ is smooth for each $l=1, \ldots, n-d$. Set $Y=H_{1} \cap \cdots \cap H_{n-d}$, which by assumption is a smooth global complete intersection in $X$ of dimension $d \geq 3$. Then a vector bundle $E$ on $X$ splits if and only if its restriction $E_{\mid Y}$ splits.

We finish with two examples whose details are easy to check.

Example 4.16. Consider the quadric surface $S:=\mathbb{P}^{1} \times \mathbb{P}^{1} \hookrightarrow \mathbb{P}^{3}=: X$ inside $\mathbb{P}^{3}$ via the Segre embedding. This is an ample surface with

$$
\mathrm{O}_{X}(S)=\mathscr{O}_{X}(2), \quad \mathscr{O}_{S}(-S)=\mathscr{O}_{S}(-1,-1),
$$

and $\Omega_{S} \cong \mathcal{O}_{S}(-2,0) \oplus \mathcal{O}_{S}(0,-2)$. Taking $E:=\Omega_{X}$ to be the rank 3 cotangent bundle on $X=\mathbb{P}^{3}$, we have the exact sequence

$$
0 \rightarrow \mathrm{O}_{S}(-1,-1) \rightarrow \Omega_{X \mid S} \rightarrow \mathrm{O}_{S}(-2,0) \oplus \mathrm{O}_{S}(0,-2) \rightarrow 0 .
$$

The obstruction to this short exact sequence splitting lies in

$\operatorname{Ext}_{\mathscr{O}_{S}}^{1}\left(\mathcal{O}_{S}(-2,0) \oplus \mathscr{O}_{S}(0,-2), \mathscr{O}_{S}(-1,-1)\right) \cong H^{1}\left(S, \mathscr{O}_{S}(1,-1)\right) \oplus H^{1}\left(S, \mathscr{O}_{S}(-1,1)\right)=0$ by the Künneth formula. Hence

$$
E_{\mid S}=\Omega_{X \mid S} \cong \mathscr{O}_{S}(-2,0) \oplus \mathcal{O}_{S}(-1,-1) \oplus \mathcal{O}_{S}(0,-2)
$$

splits on a smooth ample surface $S$ in $X=\mathbb{P}^{3}$, but $E=\Omega_{\mathbb{P}^{3}}$ itself does not split over $\mathbb{P}^{3}$ since $H^{1}\left(\mathbb{P}^{3}, \Omega_{\mathbb{P}^{3}}\right) \cong \mathbb{C} \neq 0$.

Example 4.17. Take $Y:=\mathbb{P}^{1} \times \mathbb{P}^{2} \subset \mathbb{P}^{2} \times \mathbb{P}^{2}=: X$ defined by the ideal $0_{X}(-1,0)$, and denote by $p_{1}$ and $q_{1}$ the projection to the first factor of $X$ and $Y$, respectively. Then $\widehat{O}_{X}(Y)=\mathscr{O}_{X}(1,0)$ is nef but not ample. Letting $E:=p_{1}^{*} \Omega_{\mathbb{P}^{2}}$, we see that $E$ is a nonsplitting rank 2 vector bundle on $X$, since by the Künneth formula $H^{1}(X, L)=0$ for any line bundle $L$ on $X$, so if $E$ were to split, we must have $H^{1}(X, E)=0$. However, by the same formula, we see that $H^{1}(X, E) \cong \mathbb{C} \neq 0$, so $E$ does not split over $X$. But

$$
E_{\mid Y} \cong q_{1}^{*}\left(\Omega_{\mathbb{P}^{2} \mid \mathbb{P}^{1}}\right) \cong \mathrm{O}_{Y}(-2,0) \oplus \mathcal{O}_{Y}(-1,0)
$$

splits over $Y$. 


\section{Acknowledgements}

The author is grateful to J. Włodarczyk for interesting discussions and to J. Wiśniewski for helpful and informative emails. The author would also like to give a heartfelt thanks to N. Mohan Kumar, who spotted an error in the first version of this paper and told the author of a class of examples of nonsplitting bundles that restrict to split bundles. The author also thanks the referee for valuable suggestions regarding the references and for sharpening the rank of the bundle in Example 4.9.

\section{References}

[Ancona et al. 1994] V. Ancona, T. Peternell, and J. A. Wiśniewski, "Fano bundles and splitting theorems on projective spaces and quadrics", Pacific J. Math. 163:1 (1994), 17-42. MR 94m:14021 Zbl 0808.14013

[Ballico 1995] E. Ballico, "A splitting criterion for rank 2 vector bundles on $\mathbb{P}^{n}$ ", Pacific J. Math. 169:1 (1995), 51-52. MR 96f:14052 Zbl 0830.14006

[Ballico 2008] E. Ballico, "A splitting criterion for vector bundles on cones over rational normal curves”, Int. J. Pure Appl. Math. 47:4 (2008), 577-580. MR 2009m:14019 Zbl 1152.14304

[Ballico and Malaspina 2008] E. Ballico and F. Malaspina, "A splitting criterion for vector bundles on blowing ups of the plane”, Int. Math. Forum 3:23 (2008), 1099-1105. MR 2009d:14056 Zbl 1159.14303

[Ballico and Malaspina 2009] E. Ballico and F. Malaspina, "Vector bundles on products of projective spaces and hyperquadrics", Rend. Istit. Mat. Univ. Trieste 41 (2009), 13-26. MR 2011h:14053 Zbl 1202.14014

[Chiantini and Madonna 2004] L. Chiantini and C. Madonna, "A splitting criterion for rank 2 bundles on a general sextic threefold”, Internat. J. Math. 15:4 (2004), 341-359. MR 2006a:14072 Zbl 1059.14054

[Costa and Miró-Roig 2005] L. Costa and R. M. Miró-Roig, "Cohomological characterization of vector bundles on multiprojective spaces”, J. Algebra 294:1 (2005), 73-96. MR 2007c:14042 Zbl 1093.14060

[Dolgachev 1982] I. Dolgachev, "Weighted projective varieties", pp. 34-71 in Group actions and vector fields (Vancouver, 1981), edited by J. B. Carrell, Lecture Notes in Math. 956, Springer, Berlin, 1982. MR 85g:14060 Zbl 0516.14014

[Grothendieck 1957] A. Grothendieck, "Sur la classification des fibrés holomorphes sur la sphère de Riemann”, Amer. J. Math. 79:1 (1957), 121-138. MR 19,315b Zbl 0079.17001

[Grothendieck 1968] A. Grothendieck, Cohomologie locale des faisceaux cohérents et théorèmes de Lefschetz locaux et globaux (SGA 2), Advanced Stud. in Pure Math 2, North-Holland, Amsterdam, 1968. MR 0476737 (57 \#16294) Zbl 0197.47202

[Hartshorne 1970] R. Hartshorne, Ample subvarieties of algebraic varieties, Lecture Notes in Mathematics 156, Springer, Berlin, 1970. MR 44 \#211 Zbl 0208.48901

[Hartshorne 1974] R. Hartshorne, "Varieties of small codimension in projective space", Bull. Amer. Math. Soc. 80 (1974), 1017-1032. MR 52 \#5688 Zbl 0304.14005

[Hartshorne 1977] R. Hartshorne, Algebraic geometry, Graduate Texts in Mathematics 52, Springer, New York, 1977. MR 57 \#3116 Zbl 0367.14001

[Horrocks 1964] G. Horrocks, "Vector bundles on the punctured spectrum of a local ring", Proc. London Math. Soc. (3) 14:4 (1964), 689-713. MR 30 \#120 Zbl 0126.16801 
[Horrocks and Mumford 1973] G. Horrocks and D. Mumford, "A rank 2 vector bundle on $\mathbb{P}^{4}$ with 15,000 symmetries”, Topology 12:1 (1973), 63-81. MR 52 \#3164 Zbl 0255.14017

[Kumar 1997] N. M. Kumar, "Construction of rank two vector bundles on $\mathbb{P}^{4}$ in positive characteristic”, Invent. Math. 130:2 (1997), 277-286. MR 98j:14055 Zbl 0913.14011

[Kumar et al. 2002] N. M. Kumar, C. Peterson, and A. P. Rao, "Construction of low rank vector bundles on $\mathbb{P}^{4}$ and $\mathbb{P}^{5}$ ", J. Algebraic Geom. 11:2 (2002), 203-217. MR 2002m:14034 Zbl 1076.14529

[Kumar et al. 2003] N. M. Kumar, C. Peterson, and A. P. Rao, "Monads on projective spaces", Manuscripta Math. 112:2 (2003), 183-189. MR 2005g:14083 Zbl 1030.14008

[Kumar et al. 2007a] N. M. Kumar, A. P. Rao, and G. V. Ravindra, "Arithmetically Cohen-Macaulay bundles on three dimensional hypersurfaces", Int. Math. Res. Not. 2007:8 (2007), Article ID rnm025. MR 2008e:14062 Zbl 1132.14040

[Kumar et al. 2007b] N. M. Kumar, A. P. Rao, and G. V. Ravindra, "Arithmetically Cohen-Macaulay bundles on hypersurfaces”, Comment. Math. Helv. 82:4(2007), 829-843. MR 2008f:14059 Zbl 1131. 14047

[Madonna 1998] C. Madonna, "A splitting criterion for rank 2 vector bundles on hypersurfaces in $\mathbb{P}^{4}$, Rend. Sem. Mat. Univ. Politec. Torino 56:2 (1998), 43-54. MR 2001m:14062 Zbl 0957.14012

[Malaspina 2008] F. Malaspina, "A few splitting criteria for vector bundles", Ric. Mat. 57:1 (2008), 55-64. MR 2009g:14051

[Okonek et al. 1980] C. Okonek, M. Schneider, and H. Spindler, Vector bundles on complex projective spaces, Progress in Mathematics 3, Birkhäuser, Boston, 1980. MR 81b:14001 Zbl 0438.32016

[Ottaviani 1989] G. Ottaviani, "Some extensions of Horrocks criterion to vector bundles on Grassmannians and quadrics", Ann. Mat. Pura Appl. (4) 155:1 (1989), 317-341. MR 91f:14018 Zbl 0718. 14010

[Ran 1983] Z. Ran, “On projective varieties of codimension 2", Invent. Math. 73:2 (1983), 333-336. MR 85g:14063 Zbl 0521.14018

[Sumihiro and Tagami 2001] H. Sumihiro and S. Tagami, "A splitting theorem for rank two vector bundles on projective spaces in positive characteristic", Hiroshima Math. J. 31:1 (2001), 51-57. MR 2001m:14063 Zbl 1064.14044

[Tango 1976] H. Tango, "On morphisms from projective space $\mathbb{P}^{n}$ to the Grassmann variety $\operatorname{Gr}(n, d)$ ", J. Math. Kyoto Univ. 16:1 (1976), 201-207. MR 53 \#5614 Zbl 0326.14015

[Wiśniewski 2002] J. A. Wiśniewski, "Uniform vector bundles on Fano manifolds and an algebraic proof of Hwang-Mok characterization of Grassmannians", pp. 329-340 in Complex geometry: collection of papers dedicated to Hans Grauert (Göttingen, 2000), edited by I. Bauer et al., Springer, Berlin, 2002. MR 2003f:14045 Zbl 1054.14056

[Zak 1993] F. L. Zak, Tangents and secants of algebraic varieties, Translations of Mathematical Monographs 127, American Mathematical Society, Providence, RI, 1993. MR 94i:14053 Zbl 0795. 14018

Received October 22, 2010. Revised November 24, 2010.

PARSA BAKHTARY

DEPARTMENT OF MATHEMATICS AND STATISTICS

King Fahd University of Petroleum and Minerals

BOX 1654

DHAHRAN 31261

SAUDi ARABIA

pbakhtary@kfupm.edu.sa

http://faculty.kfupm.edu.sa/MATH/pbakhtary 


\title{
PACIFIC JOURNAL OF MATHEMATICS
}

\author{
http://www.pjmath.org \\ Founded in 1951 by
}

E. F. Beckenbach (1906-1982) and F. Wolf (1904-1989)

\section{EDITORS}

V. S. Varadarajan (Managing Editor)

Department of Mathematics

University of California

Los Angeles, CA 90095-1555

pacific@math.ucla.edu

Vyjayanthi Chari

Department of Mathematics

University of California

Riverside, CA 92521-0135

chari@math.ucr.edu

\section{Robert Finn}

Department of Mathematics Stanford University

Stanford, CA 94305-2125

finn@math.stanford.edu

Kefeng Liu

Department of Mathematics

University of California

Los Angeles, CA 90095-1555

liu@math.ucla.edu
Darren Long

Department of Mathematics

University of California

Santa Barbara, CA 93106-3080

long@math.ucsb.edu

Jiang-Hua Lu

Department of Mathematics

The University of Hong Kong

Pokfulam Rd., Hong Kong jhlu@maths.hku.hk

Alexander Merkurjev

Department of Mathematics University of California

Los Angeles, CA 90095-1555 merkurev@math.ucla.edu
Sorin Popa

Department of Mathematics

University of California

Los Angeles, CA 90095-1555

popa@math.ucla.edu

Jie Qing

Department of Mathematics

University of California

Santa Cruz, CA 95064

qing@ cats.ucsc.edu

Jonathan Rogawski

Department of Mathematics

University of California

Los Angeles, CA 90095-1555

jonr@math.ucla.edu

\section{PRODUCTION}

pacific@math.berkeley.edu

Silvio Levy, Scientific Editor Matthew Cargo, Senior Production Editor

ACADEMIA SINICA, TAIPEI

CALIFORNIA INST. OF TECHNOLOGY

INST. DE MATEMÁTICA PURA E APLICADA

KEIO UNIVERSITY

MATH. SCIENCES RESEARCH INSTITUTE

NEW MEXICO STATE UNIV.

OREGON STATE UNIV.

\section{SUPPORTING INSTITUTIONS}

STANFORD UNIVERSITY
UNIV. OF BRITISH COLUMBIA
UNIV. OF CALIFORNIA, BERKELEY
UNIV. OF CALIFORNIA, DAVIS
UNIV. OF CALIFORNIA, LOS ANGELES
UNIV. OF CALIFORNIA, RIVERSIDE
UNIV. OF CALIFORNIA, SAN DIEGO
UNIV. OF CALIF., SANTA BARBARA

UNIV. OF CALIF., SANTA CRUZ

UNIV. OF MONTANA

UNIV. OF OREGON

UNIV. OF SOUTHERN CALIFORNIA

UNIV. OF UTAH

UNIV. OF WASHINGTON

WASHINGTON STATE UNIVERSITY

These supporting institutions contribute to the cost of publication of this Journal, but they are not owners or publishers and have no responsibility for its contents or policies.

See inside back cover or www.pjmath.org for submission instructions.

The subscription price for 2011 is US \$420/year for the electronic version, and \$485/year for print and electronic.

Subscriptions, requests for back issues from the last three years and changes of subscribers address should be sent to Pacific Journal of Mathematics, P.O. Box 4163, Berkeley, CA 94704-0163, U.S.A. Prior back issues are obtainable from Periodicals Service Company, 11 Main Street, Germantown, NY 12526-5635. The Pacific Journal of Mathematics is indexed by Mathematical Reviews, Zentralblatt MATH, PASCAL CNRS Index, Referativnyi Zhurnal, Current Mathematical Publications and the Science Citation Index.

The Pacific Journal of Mathematics (ISSN 0030-8730) at the University of California, c/o Department of Mathematics, 969 Evans Hall, Berkeley, CA 94720-3840, is published monthly except July and August. Periodical rate postage paid at Berkeley, CA 94704, and additional mailing offices. POSTMASTER: send address changes to Pacific Journal of Mathematics, P.O. Box 4163, Berkeley, CA 94704-0163.

PJM peer review and production are managed by EditFLOW ${ }^{\mathrm{TM}}$ from Mathematical Sciences Publishers.

PUBLISHED BY PACIFIC JOURNAL OF MATHEMATICS

at the University of California, Berkeley 94720-3840

A NON-PROFIT CORPORATION

Typeset in IATEX

Copyright $(2011$ by Pacific Journal of Mathematics 


\section{PACIFIC JOURNAL OF MATHEMATICS}

Volume $252 \quad$ No. $1 \quad$ July 2011

Some dynamic Wirtinger-type inequalities and their applications

RaVi P. Agarwal, Martin Bohner, Donal O'REgAn and SAMIR H.

SAKER

Splitting criteria for vector bundles on higher-dimensional varieties

PARSA BAKHTARY

Average Mahler's measure and $L_{p}$ norms of unimodular polynomials

KWOK-KWOng STEPHen CHOI and Michael J. MossinghofF

Tate resolutions and Weyman complexes

David A. CoX and EVgeny Materov

On pointed Hopf algebras over dihedral groups

FERNANDO FANTINO and GASTON ANDRÉS GARCIA

Integral topological quantum field theory for a one-holed torus

PATRicK M. Gilmer and Gregor Masbaum

Knot 4-genus and the rank of classes in $\boldsymbol{W}(\mathbb{Q}(t))$

CHARLES LIVINGSTON

Roots of Toeplitz operators on the Bergman space

ISSAM LOUHICHI and NAGISETTY V. RAO

Uniqueness of the foliation of constant mean curvature spheres in asymptotically 145 flat 3-manifolds

SHIGUANG MA

On the multiplicity of non-iterated periodic billiard trajectories

MARCo MAZZUCCHELLI

A remark on Einstein warped products

MiCHELE RIMOLDI

Exceptional Dehn surgery on large arborescent knots

YING-QING WU

Harnack estimates for the linear heat equation under the Ricci flow 